\title{
El modo infinito que es un individuo: la faz de todo el universo en la filosofía de Spinoza
}

\section{The infinite mode that an individual is: the face of the whole universe in Spinoza's philosophy}

\author{
CLAUDIA AGUILAR \\ Universidad de Buenos Aires / CONICET
}

Recibido: 06/06/2020 Aceptado:24/06/2020

\section{RESUMEN}

El análisis de los modos infinitos spinozianos ha sido muy variado. Mi intención es resaltar la pertinencia de los modos infinitos sin negar su peculiaridad, es decir, sin confundirlos tanto con los modos finitos como con la sustancia. La hipótesis que me interesa defender es, en primer lugar, que los modos infinitos son eternos y no sempiternos. En segundo lugar, que los modos infinitos, en particular la faz de todo el universo, son imprescindibles a la hora de pensar las relaciones parte-todo, sustancia-modos, sustancia-modos finitos e, incluso, las relaciones intramodales.

\section{PALABRAS CLAVE}

SPINOZA, INDIVIDUO, MODOS INFINITOS, FAZ DE TODO EL UNIVERSO

\section{ABSTRACT}

The analysis of the Spinozian infinite modes has been extremely varied. This article intends to highlight the relevance of infinite modes without denying their peculiarity, that is, without confusing them with both finite modes and substance. My hypothesis is, firstly, that infinite modes are eternal and not sempiternal. Secondly, that infinite modes, particularly the face of the whole universe, are indispensable to consider the relationships part-whole, substance-modes, substance - finite modes, and even intramodal relationships.

KEYWORDS

SPINOZA, INDIVIDUAL, INFINITE MODES, FACE OF THE WHOLE UNIVERSE

(C) Contrastes. Revista Internacional de Filosofía, vol. XXVI No2 (2021), pp. 7-23. ISSN: 1136-4076 
As Boxes small, \& smaller may containe, So bigger, and bigger must there be again.

Infinite may run contracting, \& dilating, Still, still, by degrees without a separating. Margareth Cavendish, Philosophicall Fancies

\section{INTRODUCCIÓN}

EL MODO INFINITO MEDiATO del atributo extensión, la faz de todo el universo, es uno de los temas sobre el que menos acuerdo hay dentro de la literatura spinozista. ${ }^{1}$ Esto se debe, a mi juicio, al poco tratamiento que hace de él nuestro filósofo. Sin embargo, considero que una de sus características lo hace llamativo: su carácter híbrido. Es un modo y es infinito. No es sustancia, pero tampoco es cosa singular. Tiene partes, pero no conatus. Es natura naturata pero no dura. Y, sobre todo, en lo que a mí me interesa, a pesar de todas estas caracterizaciones una cosa es clara: es mencionado como un individuo por Spinoza. Así, ya se puede divisar la complejidad que trae aparejada el análisis de los modos infinitos y del modo infinito mediato del atributo extensión: la faz de todo el universo. El modo infinito mediato es el foco en el que confluyen muchas nociones: sustancia, atributo, modo, cosa singular, conatus, individuo. Explicarlo, en parte, también será explicarlas a ellas.

El análisis de los modos infinitos ha sido muy variado. En general se ha desestimado su importancia, o bien, se ha resaltado su relevancia pero negando la diferencia con los modos finitos. ${ }^{2} \mathrm{Mi}$ intención, en cambio, es resaltar la pertinencia de los modos infinitos ${ }^{3}$ sin negar su peculiaridad, es decir, sin confundirlos tanto con los modos finitos como con la sustancia. La

1 Me refiero aquí al atributo extensión porque el análisis surge a partir de la digresión física. Ahora bien, considerando la correlación de atributos de la filosofía spinoziana, lo mismo vale para el atributo pensamiento: el modo infinito mediato del atributo pensamiento es la totalidad de la que todas las ideas son parte. Así lo sostiene Elisabeth Schmitt (Cf Schmitt 1910, p. 104.); más allá del problema nominal de cómo llamar a esta totalidad. Esto es así porque un modo de la extensión y un modo del pensamiento son una sola y misma cosa pero expresada de dos maneras ( $C f$. E II, prop. 7, esc.). Aunque en realidad según dicha proposición la mismidad abarcaría cualquier atributo de los cuales solo conocemos dos.

2 Este es el caso, por ejemplo, del artículo «Spinoza’s ‘Infinite Modes' Reconsidered» de Kristin Primus (Cf. Primus 2019).

3 El carácter de suma importancia de los modos infinitos en la filosofía spinoziana ha sido resaltado por Elizabeth Schmitt, quien dedica un libro entero a estos: Die unendlichen Modi bei 
hipótesis que me interesa defender es, en primer lugar, que los modos infinitos son eternos y no sempiternos. ${ }^{4}$ En segundo lugar, que los modos infinitos, en particular la faz de todo el universo, son imprescindibles a la hora de pensar las relaciones parte-todo, sustancia-modos, sustancia-modos finitos e, incluso, las relaciones intramodales. Y que, por lo tanto, eludir estos modos es inadmisible.

Respecto de la faz de todo el universo (que es el modo infinito mediato del atributo extensión y que es un individuo), tanto la digresión física como la carta 32 nos dejan el mismo problema: ¿cómo entender un individuo que es infinito? En la digresión física el escolio del lema 7 postula que «toda la naturaleza es un solo individuo, cuyas partes - esto es, todos los cuerposvarían de infinitas maneras sin cambio alguno del individuo total». ${ }^{5}$ Aquí aparece un primer problema: se habla de naturaleza y de partes. Sin embargo, lo anterior parecería entrar en contradicción con la proposición 13 de Ética I que sostiene que la «sustancia absolutamente infinita es indivisible». ${ }^{6}$ Por lo tanto, esa naturaleza del escolio del lema 7 cuyas partes son todos los cuerpos no puede ser la sustancia. Qué es dicha naturaleza que es un individuo total lo va a explicar Spinoza brevemente en el epistolario, cuando vuelve a referirse a este individuo en la carta 32 y cuando nombra ese individuo en la carta 64. El recorrido entonces será primero explorar por qué la sustancia no es este individuo total, o por qué no puede ser individuo alguno. En segundo lugar caracterizar los modos infinitos y, acto seguido, cómo es este individuo infinito tanto en la Ética como en la correspondencia de Spinoza. Por último, abordaré los problemas y contradicciones que genera la faz de todo el universo dentro de la filosofía spinoziana.

Spinoza. (Cf. Schmitt 1910, p. 121). La autora también considera que en los modos infinitos se encuentra el principio de individuación (Cf. Ibid., pp. 119-120).

4 Me distancio en este punto de Melamed quien considera a los modos infinitos sempiternos (Cf. Melamed 2013, p. 127. Ver en particular el capítulo «The Infinite Modes»). Asimismo Schmaltz también afirma la hipótesis de que el modo infinito mediato tiene duración sempiterna (Cf. Schmaltz 1997, p. 210).

5 E II, prop. 13, lem. 7. Para citar la Ethica ordine geometrico demonstrata, como es habitual, utilizo la abreviatura $\mathrm{E}$, seguida de la parte con números romanos y las siguientes abreviaturas y números arábigos para indicar las diferentes secciones: def. (definición), ax. (axioma), prop. (proposición), lem. (lema), post. (postulado), esc. (escolio), cor. (corolario), ap. (apéndice), pref. (prefacio), def. gral. de los af. (definición general de los afectos). Asimismo cito según la traducción castellana de Vidal Peña detallada en la bibliografía.

6 E I, prop. 13. 


\section{II. ¿POR QUÉ LA SUSTANCIA NO ES UN INDIVIDUO?}

La primera parte de la Ética caracteriza de manera detallada la única sustancia. Una de las características de la sustancia más desarrolladas allí es que la sustancia no tiene partes, por ejemplo, cuando Spinoza sostiene: «una sustancia absolutamente infinita es indivisible». ${ }^{7}$ La sustancia no es un individuo por este motivo, por no tener partes — no porque sea infinita, causa sui o eterna. ${ }^{8}$ De hecho, nada impide en la definición de individuo que éste pueda ser infinito o eterno. Si tomamos en consideración lo anterior con la definición de individuo que encontramos en la digresión física como una relación de partes, ${ }^{9}$ vemos claramente que ambos conceptos se excluyen. Sin embargo, otras afirmaciones spinozianas prestan lugar a confusiones.

El sentido de naturaleza que usa Spinoza en el escolio del lema 7 anteriormente citado es ambiguo; y, por lo tanto, continúa siendo un riesgo confundir la sustancia con el modo infinito mediato, sobre todo, porque Spinoza varias veces llama a este último simplemente «naturaleza». Así, por ejemplo, cuando explica las pasiones en la cuarta parte, Spinoza sostiene: «padecemos en la medida en que somos una parte de la naturaleza que no puede concebirse

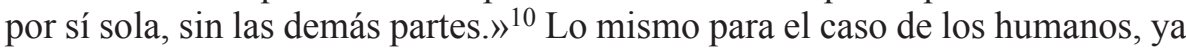
que es imposible que no sean una parte de la naturaleza y que no puedan sufrir cambios más que los inteligibles por su sola naturaleza. ${ }^{11}$ De igual manera cuando afirma que: «ningún mal puede sobrevenirle al hombre sino en virtud de causas exteriores; es decir, en cuanto es una parte de la naturaleza total, a cuyas leyes está obligada a obedecer la naturaleza humana, acomodándose prácticamente de infinitas maneras a dicha naturaleza total»». ${ }^{12}$ Vemos que confluyen términos que se excluyen por definición si consideramos que esta naturaleza total es la sustancia como natura naturans, porque la sustancia en cuanto sustancia no puede tener partes. La sustancia no es un todo porque no tiene partes. Desambiguar, entonces, qué es esta naturaleza total es el objetivo de los próximos dos apartados. ${ }^{13}$ Para lo anterior, será necesario primero

7 E I, prop. 13. Así también en E I, prop. 15, esc.

8 En este punto mi lectura se distancia de la de Sarah Kizik quien considera que la imposibilidad de la sustancia de ser un individuo se debe a que esta es causa de sí ( $C f$. Kizuk 2014, p. 42).

9 E II, prop. 13, def.

10 E IV, prop. 2.

11 E IV, prop. 4.

12 E IV, cap 6. La cursiva es un agregado mío.

13 Sobre la importancia de este tema sostiene Maria Luísa Ribeiro Ferreira: «Se fizermos uma pesquisa na literatura espinosana verificamos que todos os grandes comentadores se debruçaram sobre este tema, sublinhando a sua dificuldade. De facto, quer o consideremos inconsistente, quer encontremos uma resposta satisfatória, não conseguimos fugir à aparente 
abordar qué entiende Spinoza por modos infinitos y cómo se caracterizan estos últimos. En segundo lugar, qué es el modo infinito mediato en el atributo extensión: la faz de todo el universo.

\section{LOS MODOS INFINITOS}

Lo primero a distinguir es la sustancia de los modos en general. En la definición de modo sostiene Spinoza: «por modo entiendo la afecciones de una sustancia, o sea, aquello que es en otra cosa, por medio de la cual es también concebido». ${ }^{14}$ Como se puede observar, los modos son modificaciones de la sustancia, sustancia en la que son y por la que son concebidos. Los modos pueden ser finitos o infinitos. Aunque Spinoza no use la expresión modos infinitos, esta nominación, además de ser la utilizada en la literatura spinozista, es una manera breve y acertada de denominar a estos modos. Al respecto de ellos, se puede sostener que los modos infinitos no tienen equivalente entre los predecesores y contemporáneos a Spinoza, ${ }^{15}$ sin embargo, son necesarios para entender la relación parte-todo, sustancia-modos, sustancia-modos finitos e, incluso, las relaciones intramodales, como mencionamos. Los modos infinitos son cruciales para entender la relación parte-todo porque la divisibilidad corresponde a los modos y no a la sustancia. ${ }^{16}$ Pero vimos que Spinoza habla de partes de la naturaleza y no de partes de la sustancia, con todas las ambigüedades que lo anterior conlleva. Para abordar los modos infinitos y comprender finalmente qué es esa naturaleza que no puede ser la sustancia es necesario analizar las proposiciones 21, 22 y 23 de la primera parte de la Ética. Proposiciones que se dedican a los modos infinitos y en las que abrevan todos los análisis de ellos.

En la proposición 21 referida a los modos infinitos inmediatos Spinoza sostiene: "Todo lo que se sigue de la naturaleza, tomada en términos absolutos, de algún atributo de Dios, ha debido existir siempre y ser infinito, o sea, es eterno e infinito en virtud de ese atributo». ${ }^{17}$ Es decir, lo que se

contradição e não conciliação de três teses simultaneamente defendidas pelo filósofo: a equivalência dos conceitos Deus, Substância e Natureza; a imutabilidade e indivisibilidade da Substância; a existência de partes da Natureza. Por muito que alguns reclamem contra a legitimidade de utilizar uma linguagem espacial quando trabalhamos os textos de Espinosa, é inegável que ele fala de «partes da natureza». Admitindo que este conceito foi ganhando conotações diferentes à medida que o seu pensamento se desenvolve, a verdade é que a expressão aparece logo nos primeiros textos, mantendo-se ao longo de toda a obra» (Ribeiro Ferreira 2012, p. 65).

14 E, I, def. 5.

15 Cf. Melamed 2013, p. 144.

16 E I, prop. 15, esc.

17 E I, prop. 21. 
sigue inmediatamente de la naturaleza de la sustancia es eterno e infinito. Spinoza equipara existir siempre y eternidad, atribuyéndole eternidad a lo que se sigue de la naturaleza tomada en términos absolutos de algún atributo de la sustancia. En este sentido, considero que sostener que los modos infinitos son sempiternos es afirmar, aunque furtivamente quizá, que los modos infinitos no son eternos. Para dar apoyo a nuestro punto, a saber, que los modos infinitos son eternos y no sempiternos (entendiendo por sempiternidad una existencia indefinida) es necesario remitirse a la definición de eternidad que encontramos en la primera parte de la Ética. En la definición 8 Spinoza sostiene:

Por eternidad entiendo la existencia misma, en cuanto se la concibe como siguiéndose necesariamente de la sola definición de una cosa eterna.

Explicación: en efecto, tal existencia se concibe como una verdad eterna, como si se tratase de la esencia de la cosa, y por eso no puede explicarse por la duración o el tiempo, aunque se piense la duración como careciendo de principio y fin.

Ya que, por cómo define Spinoza los modos infinitos (lo que se sigue de la naturaleza tomada en términos absolutos de algún atributo de la sustancia) en la proposición 21 estos han debido existir siempre, su existencia se sigue necesariamente de la sustancia y por lo tanto «no puede explicarse por la duración o el tiempo, aunque se piense la duración como careciendo de principio y fin». En otras palabras, los modos infinitos no pueden ser sempiternos. Y esto, sobre todo, si consideramos la propia distinción que realiza Spinoza entre eternidad y duración sin principio ni fin, sumado al hecho de que explícitamente caracteriza los modos infinitos como eternos en la proposición mencionada. ${ }^{18}$ Sostener la sempiternidad de los modos mitiga tanto la caracterización de eternidad de los modos que encontramos en el texto como la propia distinción spinoziana entre eternidad y duración.

Así también sucede con los modos que se siguen de los modos inmediatos a la sustancia. Encontramos en la proposición 22 de Ética I: «Todo lo que se sigue a partir de un atributo de Dios, en cuanto afectado de una modificación tal que en virtud de dicho atributo existe necesariamente y es infinita, debe

18 Sobre este punto sostiene Guillermo Sibilia: «Para algunos intérpretes, resulta imposible calificar de eternos a los modos infinitos: en efecto, si la eternidad es la propiedad de aquello que existe por sí, esta modalidad de existencia no puede pertenecer a los modos (que existen por otro) sino exclusivamente a la Naturaleza Naturante. Esto ha llevado a esos intérpretes a pensar que la eternidad de los modos infinitos no es en realidad sino una existencia sempiterna o una duración infinita, o bien a considerar que Spinoza no es del todo riguroso en la presentación de su filosofía. Sin embargo, hay que tomar muy en serio el hecho de que Spinoza afirme explícitamente - y que demuestre en una proposición — que los modos infinitos son 'eternos' (E I, 21-23).» Sibilia 2017, p. 243. Agradezco al autor los comentarios al presente trabajo. 
también existir necesariamente y ser infinito». ${ }^{19}$ Así la modificación que se sigue de la modificación inmediata de la sustancia también es eterna e infinita, no sempiterna. En efecto, la eternidad abarca tanto a los modos infinitos inmediatos como a los modos infinitos que se siguen de estos, es decir, los mediatos.

La última proposición que aborda los modos infinitos excluye la posibilidad de otros modos infinitos que no se expliquen por las dos proposiciones anteriores:

Todo modo que existe necesariamente y es infinito, ha debido seguirse necesariamente, o bien de la naturaleza de algún atributo de Dios considerada en absoluto, o bien a partir de algún atributo afectado de una modificación que existe necesariamente y es infinita. ${ }^{20}$

Los modos infinitos son modificaciones de la sustancia, modos infinitos eternos que efectivamente expresan la potencia de la sustancia. Por un lado, Spinoza nunca dice que solo la sustancia es eterna, ${ }^{21}$ aunque sí es la sustancia la que aparece caracterizada como eterna en una gran cantidad de ocasiones. No obstante, también es preciso decir que los modos infinitos son caracterizados como eternos tan pronto como Spinoza se explaya sobre ellos.

Los modos infinitos son infinitos en virtud de su propia causa y no de sí mismos, ${ }^{22}$ y pueden ser inmediatos o mediatos. El efecto de un modo infinito no puede ser un modo finito. ${ }^{23}$ Ahora bien, los modos infinitos no son infinitos por ser modos, ya que los hay finitos e infinitos, sino por su relación con la sustancia. Lo anterior nos lleva a la distinción entre natura naturans (naturaleza naturante) y natura naturata (naturaleza naturada). Sostiene Spinoza en el escolio a la proposición 29 de Ética I:

Antes de seguir adelante, quiero explicar aquí —o más bien advertir- qué debe entenderse por Naturaleza naturante y qué por Naturaleza naturada. Pues

19 E I, prop. 22

20 E I, prop. 23.

21 Cf. Primus 2019, p. 17.

$22 \mathrm{Al}$ respecto señala Mariana de Gainza en «El problema del infinito en Spinoza. El arte de la distinción»: «hay que saber distinguir, en primer lugar, la cosa que es infinita en virtud de su propia esencia: la sustancia única absolutamente infinita y la infinidad de atributos infinitos en su género que la constituyen. En este sentido, el infinito es una de las propiedades (junto con la eternidad, la simplicidad, la indivisibilidad) del ser cuya esencia envuelve la existencia necesaria [...]. En segundo lugar, hay que saber distinguir las cosas que son infinitas en virtud de la causa de la que dependen: se trata de los modos infinitos, inmediatos y mediatos, en los cuales los atributos se expresan necesariamente.» Gainza 2017 (en prensa).

23 E I. prop. 22. 
creo que ya consta, por lo anteriormente dicho, que por Naturaleza naturante debemos entender lo que es en sí y se concibe por sí, o sea, los atributos de la sustancia que expresan una esencia eterna e infinita, esto es (por el Corolario de la Proposición 14 y el Corolario 2 de la Proposición 17), Dios, en cuanto considerado como causa libre. Por Naturaleza naturada, en cambio, entiendo todo aquello que se sigue de la necesidad de la naturaleza de Dios, o sea, de cada uno de los atributos de Dios, esto es, todos los modos de los atributos de Dios, en cuanto considerados como cosas que son en Dios, y que sin Dios no pueden ser ni concebirse. ${ }^{24}$

Si la natura naturans es indivisible, autocausada y se concibe por sí, los modos infinitos, en tanto modos, son natura naturata. Sin embargo, no duran. ${ }^{25}$ La esencia de la sustancia y de los modos infinitos no es el conatus, el esfuerzo por perseverar en el ser, porque el conatus se relaciona con la duración. Según E III, prop. 8, el conatus implica tiempo indefinido por lo que la sustancia y los modos infinitos no pueden tener conatus. ${ }^{26}$

A raíz de las proposiciones mencionadas de la primera parte de la Ética $(21,22$ y 23), Spinoza recibe en una carta un pedido de aclaración por parte de uno de sus corresponsales en 1675. La carta es de Georg Hermann Schuller, médico alemán que estudió en Leiden y se radicó en Amsterdam. La actitud de Schuller lo muestra bastante absorto y su pedido es el siguiente:

Desearía ejemplos de aquellas cosas que son inmediatamente producidas por Dios y de aquellas que lo son mediante alguna modificación infinita. A mí me parece que son del primer género el pensamiento y la extensión, y del segundo, el entendimiento en el pensamiento, y el movimiento, en la extensión, etc. ${ }^{27}$

Spinoza responde al pedido en la carta 64 negando, en parte, la nominación que había ofrecido Schuller y que generaba una homonimia entre los modos infinitos inmediatos y los atributos. Contesta Spinoza:

24 E I, prop. 29, esc.

25 Sobre la existencia y la duración afirma Spinoza: «Por 'existencia' no entiendo aquí la duración, esto es, la existencia en cuanto concebida abstractamente y como si fuese una especie de cantidad.» E II, prop. 45, esc.

26 Si la sustancia tiene o no conatus es una discusión dentro del spinozismo. Una interpretación contraria a la propuesta aquí se encuentra, por ejemplo, en el trabajo mencionado de Sarah Kizuk, cuando sostiene que todas las cosas tienen conatus. (Cf. Kizuk 2014, pp. 4546). Pero, a mi juicio, ni los cuerpos simples tienen conatus ni la faz de todo el universo, ni los modos infinitos, ni la sustancia.

27 Ep. 63: 276. Trad. cast.: 348. Cito según la traducción de Domínguez mencionada en la bibliografía. Asimismo, primero indico el número de carta tras la sigla Ep. y remito a la página de la edición canónica de los textos de Spinoza realizada por Carl Gebhardt referida en la bibliografía. Ep. 30, p. 166. Trad. cast.: 231. 
Finalmente, los ejemplos que usted pide son: del primer género, en el pensamiento, el entendimiento absolutamente infinito; en la extensión, en cambio, el movimiento y el reposo; del segundo género, la faz de todo el universo, la cual, aunque varíe de infinitos modos, permanece, no obstante, siempre la misma: vea sobre esto el escolio del lema 7 que precede a la proposición 14 de la parte II. ${ }^{28}$

Es decir, al ser consultado sobre los modos infinitos, Spinoza remite al escolio nombrado para explicar el modo finito mediato del atributo extensión, que nomina «faz de todo el universo». Así podemos sostener que lo que en el escolio es presentado como la naturaleza total o el individuo del que todos los cuerpos son parte es lo que en la carta anterior aparece como modo finito mediato: faz de todo el universo. Todo remite a la misma cosa. Spinoza responde de una manera vertiginosa y fugaz. Los modos infinitos inmediatos son el intelecto absolutamente infinito en el atributo pensamiento y movimiento y reposo en el atributo extensión. El modo infinito mediato en el atributo extensión es la faz de todo el universo. Un primer problema surge por no tener nombrado el modo infinito mediato del atributo pensamiento. Sin embargo, esto no genera un desfasaje en la correlación de atributos. ${ }^{29}$ En lo que a nuestro tema respecta, resta dedicarnos a aquel modo infinito que es un individuo según el escolio del lema al que Spinoza remite en la carta 64.

\section{EL INDIVIDUO INFINITO, LA FAZ DE TODO EL UNIVERSO, EN LA ÉTICA Y EN LAS CARTAS}

Si bien, considerando los dos atributos que conocemos, habría cuatro modos infinitos (aunque de uno parece no haber una denominación específica), me voy a centrar en el que aparece explícitamente nombrado en la digresión física como individuo: la faz de todo el universo. En el mencionado escolio del último lema de la digresión física —al que Spinoza remite en la sombría

28 Ep. 64: 278. Trad. cast.: 351.

29 Otra interpretación que ha surgido, y que no compartimos, es aquella que considera que no falta el nombre del modo infinito mediato del atributo pensamiento, sino que la faz de todo el universo corresponde tanto al atributo extensión como al atributo pensamiento. ( $C f$. Dujovne 2015, Tomo II, p. 84). En la proposición 31 de la misma parte Spinoza habla de un entendimiento infinito en acto que pertenece a la naturaleza naturada. Este último podría ser un buen candidato para llenar el hueco que Spinoza deja en la carta. Esta es la interpretación, por ejemplo, de Vidal Peña ( $C f$. Spinoza, Baruch, Ética demostrada según el orden geométrico, trad. por V. Peña, Madrid, Editora Nacional, 1980, nota 18), quien asimismo desarrolla los problemas de considerar la caracterización «en acto» de este entendimiento. Sin embargo, persiste la inquietud de por qué Spinoza no lo caracterizó como un modo infinito ni lo nombró como tal en la respuesta a su corresponsal. Sea como sea, el nombre del modo infinito mediato del atributo pensamiento no genera un cambio en el análisis del presente trabajo. 
respuesta a Schuller ya citada - encontramos después de referirse a los cuerpos simples y a los individuos de primer y segundo grado de individualidad:

Si concebimos, además, un tercer género de individuos, compuesto de individuos del segundo género, hallaremos que puede ser afectado de otras muchas maneras, sin cambio alguno de su forma. Y si continuamos así hasta el infinito, concebiremos que toda la naturaleza es un solo individuo, cuyas partes - esto es, todos los cuerpos - varían de infinitas maneras sin cambio alguno del individuo total. $^{30}$

En el apartado II ya fue dilucidado que lo que aparece como naturaleza en este escolio no puede ser la sustancia porque la sustancia jamás podría ser considerada un individuo. Según dicha carta, el escolio del lema 7 se refiere al modo infinito mediato del atributo extensión: la faz de todo el universo. Esta faz de todo el universo es lo que da unidad a todos los cuerpos finitos, los entrelaza, cuerpos finitos que pasan a ser partes de este individuo, partes que en tanto tales concuerdan entre sí. Los modos finitos conforman el modo infinito mediato en tanto que partes de éste, de este todo que también caracteriza Spinoza como «el conjunto». Al respecto cabe considerar la carta 32. Allí Spinoza brinda el ejemplo del gusano y la sangre para explicar la relación de las partes con el todo y de las partes entre sí. ${ }^{31}$ Luego señala:

Ahora bien, todos los cuerpos de la naturaleza pueden y deben ser concebidos del mismo modo que acabamos de concebir la sangre, puesto que todos ellos están rodeados por otros y se determinan mutuamente a existir y a obrar de una forma segura y determinada, de suerte que, al mismo tiempo, se mantenga siempre constante en el conjunto, es decir, en todo el universo, la misma proporción entre el movimiento y el reposo. De donde se sigue que todo cuerpo, en cuanto que esté modificado de alguna manera, debe ser considerado como una parte de todo el universo, y debe estar acorde con su todo y en conexión con los demás cuerpos. Pero, como la naturaleza del universo no es limitada, como la naturaleza de la sangre, sino absolutamente infinita, sus partes son de mil maneras moduladas por esa naturaleza de poder infinito y son forzadas a sufrir infinitas variaciones. ${ }^{32}$

La faz de todo el universo es el conjunto constante cuyas partes varían continuamente. Siendo este el panorama, se puede sostener la segunda parte de nuestra hipótesis en el presente capítulo: los modos infinitos no son algo omitible de la filosofía spinoziana, ${ }^{33}$ al ser justamente lo que explica la

30 E II, prop. 13, lem. 7.

$31 \mathrm{Al}$ respecto, puede consultarse «La razón del gusano: mereología en la filosofía de Spinoza» (Aguilar 2020).

32 Ep. 32. pp. 172-173. Trad. cast.: 237.

33 Sobre la importancia de los modos infinitos sostiene Peterman: «Finally, drawing on 
causalidad no transitiva de la sustancia; y, en el caso de la faz de todo el universo, lo que aúna todos los cuerpos finitos. La completa evasión de los modos infinitos en el análisis esta filosofía spinoziana traería problemas en las implicancias que Spinoza saca a partir de ellos. Por ejemplo, una consecuencia de las proposiciones 21 a 23 de la primera parte de la Etica, que se refieren a los modos infinitos, se puede observar en el escolio a la proposición 28 de la misma parte:

Como ciertas cosas han debido ser producidas por Dios inmediatamente, a saber: las que se siguen necesariamente de la naturaleza considerada en absoluto, $y$, por la mediación de estas primeras, otras, que, sin embargo, no pueden ser ni concebirse sin Dios, se sigue de aquí: primero, que Dios es causa absolutamente «próxima» de las cosas inmediatamente producidas por él; y no «en su género», como dicen. Se sigue: segundo, que Dios no puede con propiedad ser llamado causa «remota» de las cosas singulares, a no ser, quizá, con objeto de que distingamos esas cosas de las que Él produce inmediatamente, o mejor dicho, de las que se siguen de su naturaleza considerada en absoluto. Pues por «remota» entendemos una causa tal que no está, de ninguna manera, ligada con su efecto. Pero todo lo que es, es en Dios, y depende de Dios de tal modo que sin Él no puede ser ni concebirse. ${ }^{34}$

Es decir, Spinoza explícitamente retoma los modos infinitos para explicar la causalidad divina de las cosas singulares, impidiendo que esta causalidad sea considerada remota. Como podemos ver, los modos infinitos se vuelven cruciales a la hora de explicar la inmanencia spinoziana evitando todo trascendencia de la sustancia respecto de los modos finitos. Otro de los riesgos de la ausencia de los modos infinitos es que los modos finitos se disuelvan en una indefinición de modos finitos, al no existir este individuo total, la faz de todo el universo, que es una ratio que siempre permanece. ${ }^{35}$ Las partes del modo infinito mediato no son partes ilusorias, sino que cada una tiene una esencia que puede conocerse adecuadamente por el tercer género de

recent work by Yitzhak Melamed, I consider the possibility that Spinoza's infinite modes can bridge the gap between Extended substance and its modes such that modes are extended in space even though substance is not.» Peterman 2015, p. 12.

34 E I, prop. 28, esc.

35 Por este motivo, Alison Peterman sostiene que Spinoza fue relacionado con la doctrina filosófica del alma del mundo, entre otros por Wachter, quien asoció a Spinoza con el alma del mundo de la Kabbalah. Según la autora, el alma del mundo tiene dos características: es un individuo unificado y explica el comportamiento de los cuerpos. $C f$. Peterman (EN PRENSA). 
conocimiento. ${ }^{36}$ Sobre la determinación de la faz de todo el universo y sus partes sostiene Deleuze:

Las diversas relaciones agrupan conjuntos infinitos variables de partes extensivas. Determinan entonces las condiciones bajo las que los modos pasan a la existencia. Cada relación efectuada constituye la forma de un individuo existente. Luego no hay relación que no se componga en otra para formar, bajo una tercera relación, un individuo de grado superior [...].

Todas las relaciones se componen al infinito para formar esa facies. Pero se componen según leyes que les son propias, leyes contenidas en el modo infinito mediato. Es decir, que las relaciones no se componen no importa cómo, cualquier relación no se compone con cualquier otra. ${ }^{37}$

La faz de todo el universo es ese individuo total en el que todas las partes concuerdan o «el conjunto» cuyas partes cambian constantemente sin tener él variación alguna, como Spinoza lo caracteriza en la carta 32.

Otro interrogante surge respecto de si acaso la faz de todo el universo es un cuerpo. Para responder a esta inquietud es necesario comenzar por la propia definición de cuerpo de la Ética. La definición de cuerpo sostiene: «Entiendo por cuerpo un modo que expresa de cierta y determinada manera la esencia de Dios, en cuanto se la considera como una cosa extensa; ver el corolario de la Proposición 25 de la Parte I». ${ }^{38}$ Según esta definición la faz de todo el universo podría ser un cuerpo porque es un modo que expresa la esencia de Dios en cuanto se la considera una cosa extensa. Pero la misma definición remite a un corolario que versa sobre cosas particulares. En el corolario de la proposición 25 de la parte I Spinoza sostiene: «Las cosas particulares no son sino afecciones de los atributos de Dios, o sea, modos por los cuales los atributos de Dios se expresan de cierta y determinada manera». ${ }^{39}$ El punto aquí es ver qué quiere decir en ese corolario «de cierta y determinada manera» para poder entender qué son las cosas particulares; Spinoza remite allí a la definición de modo ${ }^{40}$ y a la proposición que afirma: «Todo lo que es, es en Dios y $\sin$ Dios nada puede ser ni concebirse. ${ }^{41}$ ¿Significa esto, acaso, que la faz de todo el universo no puede ser un cuerpo? Ciertamente, no. Nada parece indicar que Spinoza equipare las cosas particulares con las cosas

36 Sigo en este punto a Melamed en su apartado dentro del capítulo dedicado a los modos infinitos «Why not 'acosmism'?» Cf. Melamed 2013.

37 Deleuze 1999, p. 227.

38 E II, def. 1

39 E I, prop. 25, corol.

40 E I, def. 5.

41 E I, prop. 15 
singulares, que son finitas y tienen una existencia limitada. ${ }^{42}$ «De cierta y determinada manera» podría ser simplemente que no incluye el otro atributo. ${ }^{43}$ Sin embargo, Spinoza también sostiene en la parte I de la Ética: «por cuerpo entendemos toda cantidad larga ancha y profunda, limitada según cierta figura». ${ }^{44}$ ¿Es realmente esta la manera en que Spinoza caracteriza al cuerpo? Considero que no. ${ }^{45}$ No es su definición, la definición de cuerpo recién vamos a encontrarla en la segunda parte de la obra. Por lo tanto, siguiendo el camino de la definición de cuerpo y los distintos lugares a los que remite, nada impide aceptar que la faz de todo el universo sea un cuerpo: el cuerpo del cual todos los otros cuerpos son partes, el único cuerpo infinito del cual son partes todos los cuerpos finitos. El problema de identificar individuo y cosa singular es que, si se parte de esta equiparación, el modo infinito mediato no podría pensarse como un individuo desde ningún punto, ya que dada su infinitud no es una cosa singular. ${ }^{46}$

Ahora bien, como ya mencioné, teniendo en cuenta que el conatus es el esfuerzo por perseverar en el ser, considero que tanto la sustancia como los modos infinitos no tienen conatus aunque sí potencia. ${ }^{47}$ Esto no afecta el carácter de individuo del modo infinito mediato, al menos no si consideramos la definición del individuo como ratio. El modo infinito mediato del atributo

42 E II, def. 7. Una lectura opuesta encontramos en León Dujovne, quien equipara cosas particulares a modos finitos. Así lo sostiene: «Vendrían ahora las cosas particulares, que podríamos designar con la letra D y de las cuales Spinoza da esta definición, en el corolario de la proposición 25 de la primera parte de la Ética: 'Las cosas particulares sólo son estados o modos de los atributos de Dios en que éstos se expresan de una manera cierta y determinada'. Spinoza no dice si se trata de modos finitos o infinitos, omisión que no es la única que hace oscura la proposición que acabamos de transcribir. Por nuestra parte admitiremos que se refiere a modos finitos» (Dujovne 2015, tomo II, p. 75).

43 En este punto mi postura se distancia de la de Alison Peterman, quien considera que todos los cuerpos son finitos equiparando esto ultimo a ser determinado. (Cf. Peterman 2015, p. 3).

44 E I, prop. 15, esc.

45 En este punto coincido con Natalia Lerussi. Véase Lerussi 2008.

46 Un ejemplo problemático de equiparación entre cosas singulares y cuerpo encontramos en el texto de Sarah Kizuk, donde la autora sostiene: «Natura Naturans is equated with Eternity, whereas we should equate natura naturata with Duration [...]. Here another complication arises. Garrett aptly points out that the 'physical interlude' shows that bodies are singular things and that singular things are shown to be finite and determinate. What can we make of the above argument that finds that the argument that an infinite mode is an individual?» Kizuk 2014, pp. 44-45. Sin embargo, lo que encontramos en la definición de la digresión física es que todo individuo es un cuerpo, no que todo cuerpo sea una cosa singular o que todo individuo sea una cosa singular.

47 Esta idea no es unánimemente aceptada por quienes se especializan en la filosofía de Spinoza. Así por ejemplo, Julie Klein sí considera que la naturaleza tiene conatus cuando afirma: «his idea of conatus or conative movement in Nature, mitigates against the viewing of Nature as complete» (Klein 2002, p. 315). 
extensión no tiene conatus, porque al ser infinito y eterno no puede tener esfuerzo por perseverar en el ser. Sin embargo, eso no quita que sea una expresión de la potencia de la sustancia, una modificación de ella.

En definitiva, la faz de todo el universo, al ser necesaria, es eterna. Asimismo es lo que aúna todos los cuerpos finitos, cuerpos finitos que concuerdan entre sí. Es esa ratio que se mantiene siempre igual a pesar de que sus partes puedan cambiar infinitamente. Es aquel todo, que sin ser parte, hace posible que todos los cuerpos finitos sean parte de una misma cosa. De esta manera, los modos infinitos, y la faz de todo el universo en particular, ayudan a la comprensión de la relación sustancia-modos, finitud-infinitud e incluso la relación intramodal que tienen todos los modos finitos respecto del modo infinito mediato del atributo correspondiente. Ahora bien, ya que todos los cuerpos tienen algo en común por ser una modificación del atributo extensión, así también las ideas de esos cuerpos; porque «el ser formal de las ideas reconoce como causa a Dios, en cuanto a este se le considera solo como cosa pensante, y no en cuanto es explicado por otro atributo». ${ }^{48}$ No obstante lo anterior, a partir de la consideración de la faz de todo el universo surgen una serie de problemas que desarrollaré a continuación.

\section{PRoblemas y CONTRADICCIONES APAREJADOS POR LA FAZ DE TODO EL UNIVERSO}

Como mencioné, la faz de todo el universo, el modo infinito mediato del atributo extensión, nos enfrenta a una serie de problemas: en primer lugar, cuando nos referimos a modos finitos la misma cosa es una parte y es un todo, parte y todo, entonces, son términos relativos ${ }^{49}$ según haya concordancia o no entre las partes. ${ }^{50}$ Esto es un inconveniente para el caso del modo infinito mediato, faz de todo el universo, que es un todo conformado por partes sin ser parte de un todo mayor. Algunas lecturas de la mereología spinoziana sostienen que todo todo es parte de un todo de mayor grado de individuación; ${ }^{51}$ sin embargo, el modo infinito mediato es un todo que no es parte. Es un todo en otro sentido. Entonces, parte y todo son términos relativos (todo todo es a su vez una parte) cuando nos manejamos en el terreno de la finitud. Sin embargo, hay distintos sentidos de «todo», no es igual para la faz de todo el universo, porque nunca es parte de un todo aunque sí sea divisible en partes finitas.

48 E II, prop. 5.

49 Cf. Sacksteder 1977, p. 143.

50 Cf. ibid., p. 141.

51 Así por ejemplo lo sostiene Sacksteder cuando afirma que la manera interrelacional en la que Spinoza define parte/todo impide suponer una parte última que no sea a la vez un todo conformado de partes y un universo total que ya no es parte de otro todo $(C f$. Sacksteder 1985, p. 397). 
Una segunda constelación de problemas entre algunas lecturas de la Ética podría surgir al considerar que los modos infinitos no están en la duración o, mejor dicho, no duran y sin embargo son natura naturata. La duración es definida en la Ética como «una continuación indefinida en la existencia».52 Esto se contrapone con la eternidad que, según mi hipótesis, Spinoza atribuye a los modos infinitos. Por este motivo, los modos infinitos (entre ellos la faz de todo el universo) no duran. Sin embargo, algunas lecturas han establecido una conexión necesaria entre natura naturata y duración, por ejemplo, sosteniendo que «la duración pertenece a existencias que tienen a Dios como su causa eficiente, es decir, pertenece a 'cosas creadas', lo que la distingue de la eternidad». ${ }^{53}$ Lo anterior es válido para los modos finitos, no lo es para los infinitos que no duran, son eternos y no por eso no son modificaciones. Este carácter híbrido de no estar en la duración y sin embargo no ser natura naturans también es un rasgo propio de los modos infinitos. Además, en el caso de la faz de todo el universo, si bien no está en la duración, sus partes sí. Los modos finitos son efectos inmanentes de la sustancia y partes de este modo infinito mediato.

La faz de todo el universo, tal como la caracterizamos, también podría generar una dificultad si tenemos en cuenta algunos puntos de la digresión física. Un inconveniente es que en la demostración del lema 3 los cuerpos aparecen como cosas singulares y allí Spinoza remite a la definición de cuerpo antes mencionada: «Los cuerpos (por la definición 1 de esta parte) son cosas singulares...» ${ }^{54}$ Es decir, si bien, como vimos, nada hay en la definición de cuerpo y en los pasajes a los que dicha definición remite que impida considerar la faz de todo el universo como un cuerpo; Spinoza equipara cuerpo y cosa singular, cuestión que sí impediría afirmar que la faz de todo el universo es un cuerpo porque claramente este modo al ser infinito no es una cosa singular. Lo que considero la salida de este terreno tan pantanoso es el hecho de que Spinoza es sumamente impreciso cuando se refiere a la noción de cosa singular. Noción que equipara a otras de su filosofía, como cuerpo o individuo, generando contradicciones al interior de dicha filosofía. En lo que a nuestro punto respecta, como vimos en el apartado anterior, no es para nada problemático sostener que la faz de todo el universo es un cuerpo si consideramos la definición de cuerpo y las partes de la Ética a las que esa noción lleva.

52 E II, def. 5. En Pensamientos metafísicos (I, 4), Spinoza define la duración de la siguiente manera: «Duración es el atributo bajo el cual concebimos la existencia de cosas creadas, en cuanto perseveran en su propia actualidad».

53 Dujovne 2015, tomo II, p. 89.

54 E II, prop. 13, lem.3, dem. 


\section{CONCLUSIONES}

Habiendo abordado los modos infinitos, vimos que estos son eternos y no sempiternos. Así también hemos apuntado a la relevancia que tienen los modos infinitos y la faz de todo el universo en particular. Detenernos en la faz de todo el universo contribuyó, a su vez, al análisis de otras nociones: sustancia, modo, eternidad, duración, cuerpo, cosa singular, conatus. Así también la faz de todo el universo es presentada como un individuo infinito que permite explorar las relaciones parte-todo, sustancia-modos, sustanciamodos finitos e, incluso, las relaciones intramodales.

Ahora bien, como observamos, la multiplicidad de los modos no daña la unidad de la sustancia, siquiera la unidad de la faz de todo el universo. La faz de todo el universo aúna todos los cuerpos finitos que pasan a ser parte de un mismo todo que los interrelaciona: el modo infinito mediato del atributo extensión. Partes que cambian continuamente en un todo en el que convergen y que permanece siempre el mismo. Así, desde los cuerpos compuestos más ínfimos, pasando por una célula, los componentes de la sangre, un órgano, un humano, un animal no humano, la tierra, el sistema solar, etc. son individuos que conforman la faz de todo el universo, también nombrada como «el conjunto», «la naturaleza toda» o «el individuo total». La faz de todo el universo permanece invariable aunque sus partes varíen constantemente. De este modo, la finitud de los individuos finitos se interrelaciona con la infinitud de la que es parte, la de los modos infinitos mediatos, en la inmanencia de la única sustancia que es a su vez infinita.

\section{REFERENCIAS BIBLIOGRÁFICAS}

AGUILAR, C. 2020: «La razón del gusano: mereología en la filosofía de Spinoza», Cadernos Espinosanos, São Paulo, vol. 43.

DELEUZE, G. 1999: Spinoza y el problema de la expresión, trad. por H. Vogel. Barcelona: Muchnik.

DUJOVNE L. 2015: Spinoza. Su vida, su época, su obra, su influencia, 4 tomos. Buenos Aires: Biblioteca Nacional.

GAINZA, M., (EN PRENSA): «El problema del infinito en Spinoza. El arte de la distinción», Ideas y Valores. Revista Colombiana de Filosofía, ${ }^{\circ}{ }^{175}$, Facultad de Ciencias Humanas de la Universidad Nacional de Colombia, Bogotá, Colombia.

KIZUK, S., 2014: Individuation and individuality: a reading of Spinoza's physical interlude. Tesis de Maestría: Memorial University of Newfoundland.

KLEIN, J., 2002: «'By Eternity I Understand': Eternity According to Spinoza», The Jerusalem Philosophical Quarterly, Nro.51, pp. 295-324.

LERUSSI, N., 2008: «Sobre la noción de cuerpo en Spinoza» en TATIÁN, D. (comp.): Spinoza. Cuarto coloquio. Córdoba: Brujas.

MELAMED,Y., 2013: Spinoza's Metaphysics Substance and Thought. Nueva York: 
OUP.

PETERMAN, A., 2015: «Spinoza on extension», Philosophers'Imprint, Nro. 15, PP. $1-23$.

PETERMAN, A., (EN PRENSA): «The world soul in early modern philosophy» en Oxford Philosophical Concepts: The World Soul.

PRIMUS, K., 2019: «Spinoza's «Infinite Modes» Reconsidered», Journal of Modern Philosophy, 1(1). DOI: http://doi.org/10.32881/jomp.69).

RIBEIRO FERREIRA, M. L., 2012: «'Um verme no sangue’ -considerações sobre a relação Todo/partes na filosofia de Espinosa», Revista de Filosofía. Universidad Iberoamericana, $\mathrm{N}^{\circ} 133$, pp. 57-81.

SACKSTEDER W., 1985: «Simple Wholes and complex Parts: Limiting principles in Spinoza», Philosophy and Phenomenological Research, Vol. 45, No. 3, Marzo.

SACKSTEDER W., 1977: «Spinoza on Part and Whole: The Worm's Eye View», The Southwestern Journal of Philosophy, Vol. 8, No. 3, Spinoza Issue, otoño, pp. 139-159.

SCHMALTZ, T., 1997: «Spinoza's Mediate Infinite Mode», Journal of the History of Philosophy 35 (2). DOI: https://doi.org/10.1353/hph.1997.0024).

SCHMITT, E., 1910: Die undendlichen Modi bei Spinoza. Leipzig: Barth.

SIBILIA, G., 2017: De la producción eterna de lo real al tiempo vivido. Ontología y temporalidad en Spinoza. Tesis doctoral, Universidad de Buenos Aires.

SPINOZA, B., 1925: Opera quae supersunt omnia, Heidelberger Akademie der Wissenschaften, editada por Carl Gebhardt, 4 tomos. Heidelberg: Carl WinterVerlag.

SPINOZA, B., 1980: Spinoza, B. Ética demostrada según el orden geométrico, trad. por V. Peña. Madrid: Editora Nacional.

SPINOZA, B., 1988: Correspondencia, trad. por A. Domínguez. Madrid: Alianza.

Claudia Aguilar es Profesora de Enseñanza Media y Superior en Filosofía y Licenciada en Filosofía por la Universidad de Buenos Aires, establecimiento en que se empeña como docente. Actualmente se encuentra finalizando su doctorado como becaria doctoral del CONICET.

Líneas de Investigación:

Baruch Spinoza y el problema del individuo en su filosofía

Publicaciones recientes:

2020: «La razón del gusano: mereología en la filosofía de Spinoza», Cadenos Espinosanos, vol. 43.

2019: Mujeres: resignación, estrategia y alianzas. Una propuesta desde la teoría de la individualidad spinoziana. Buenos Aires: RAGIF Ediciones.

Correo electrónico: claudiamaaguilar@gmail.com 
